\title{
Experimental study of the effects of the spatial filtering on off-axis digital holography operating out and in of the diffraction limit
}

\section{Estudio experimental de los efectos del filtrado espacial en holografía digital fuera de eje operando fuera o dentro en el límite de difracción}

\author{
Raúl Castañeda ${ }^{1}$, Diego Hincapié-Zulauga ${ }^{1,2}$, Jorge Garcia-Sucerquia1 ${ }^{1,}$ \\ 1. Universidad Nacional de Colombia sede Medellín, Escuela de Física - A.A: 3840-Medelin-050034-Colombia \\ 2. Instituto tecnológico metropolitano (ITM), Medellín, Colombia, 050034 \\ ${ }^{(*)}$ E-mail: jigarcia@unal.edu.co
}

Received: 21/10/2016 Accepted: 20/01/2017

DOI: $10.7149 / 0 P A .50 .1 .49016$

\begin{abstract}
:
This paper presents a theoretical and experimental analysis of the close relationship between the recording of digital holograms operating out or in of the diffraction limit and the spatial filtering process in the overall performance of the numerical reconstruction of the digitally recorded holograms. The results allow to conclude that it is not possible to make a correct spatial filtering for a reliable numerical reconstruction of the recorded holograms, if the recording is not made in the diffraction limit or presents overlapping of the diffraction orders.
\end{abstract}

Key words: Digital holography, spatial filter, diffraction limit.

\section{RESUMEN:}

En este trabajo se hace un análisis teórico y experimental de la estrecha relación que existe entre el registro de hologramas digitales operando o no en el límite de difracción y del proceso de filtrado espacial en el desempeño global de la reconstrucción numérica de hologramas registrados digitalmente. Los resultados obtenidos permiten concluir que no es posible hacer un filtrado espacial correcto del holograma para su posterior reconstrucción numérica, si el registro de holograma no es realizado en el límite de difracción o en su defecto sin solapamiento de los órdenes de difracción.

Palabras clave: Holografía digital, filtrado espacial, límite de difracción.

\section{REFERENCES AND LINKS / REFERENCIAS Y ENLACES}

[1] U. Schnars and W. Jüptner, "Direct recording of holograms by a CCD target and numerical reconstruction.", Appl. Opt. 33, 179-81 (1994). https://doi.org/10.1364/A0.33.000179

[2] M. K. Kim, Digital Holographic Microscopy. Principles, techniques, and Aplications, Springer, (2011). https://doi.org/10.1007/978-1-4419-7793-9

[3] A. Asundi, Digital Holography for MEMS and Microsystem Metrology, (2011).

[4] O. Matoba and B. Javidi, "Encrypted optical memory system using three-dimensional keys in the Fresnel domain", Opt. Lett. 24, 762-764 (1999). https://doi.org/10.1364/OL.24.000762

[5] J. W. Goodman, Statistical Optics, Wiley, (1985).

[6] D. Hincapie, J. Herrera-Ramírez, J. Garcia-Sucerquia, J. Herrera-Ramirez, and J. Garcia-Sucerquia, "Single-shot speckle reduction in numerical reconstruction of digitally recorded holograms", Opt. 
Lett. 40, 1623-1626 (2015).

https://doi.org/10.1364/OL.40.001623

[7] J. Garcia-Sucerquia, J. H. Ramírez, R. Castaneda, J. Herrera-Ramírez, and R. Castaneda, "Incoherent recovering of the spatial resolution in digital holography", Opt. Commun. 260, 62-67 (2006). https://doi.org/10.1016/i.optcom.2005.10.003

[8] J. Garcia-Sucerquia, J. H. Ramírez, and D. Velasquez Prieto, "Reduction of spleckle noise in digital holography by using digital image processing", Opt. J. Light Electron Opt. 116, 44-48 (2005). https://doi.org/10.1016/i.ijleo.2004.12.004

[9] J. W. Goodman, Introduction to Fourier Optics, Roberst \& Company Publishers, Greenwood Village, Colo, (2005).

[10] T. Kreis, Handbook of Holographic Interferometry: Optical and Digital Methods, Wiley-vch Verlag Ed, Weinheim, Weinheim, (2005).

[11] O. K. Ersoy, Diffraction, Fourier Optics and Imaging, Hobenken, New Jersey, (2006).

[12] J. Li and P. Picart, "Calculating Diffraction by Fast Fourier Transform", Digit. Hologr. 77-114 (2012).

[13] T. Kreis, Handbook of Holographic Interferometry, Wiley-VCH Verlag GmbH \& Co. KGaA, Weinheim, FRG, (2004).

[14] J. F. Restrepo and J. Garcia-Sucerquia, "Magnified reconstruction of digitally recorded holograms by Fresnel-Bluestein transform.", Appl. Opt. 49, 6430-6435 (2010). https://doi.org/10.1364/A0.49.006430

[15] D. Mendlovic, Z. Zalevsky, and N. Konforti, "Computation considerations and fast algorithms for calculating the diffraction integral", J. Mod. Opt. 44, 407-414 (1997). https://doi.org/10.1080/09500349708241880

[16] M. Sypek, C. Prokopowicz, and M. Go'recki, "Image multiplying and high-frequency oscillations effects in the Fresnel region light propagation simulation", Opt. Eng. 42, 3158-3164 (2003). https://doi.org/10.1117/1.1613959

[17] R. Castañeda, W. Toro, and J. Garcia-Sucerquia, "Evaluation of the limits of application for numerical diffraction methods based on basic optics concepts", Opt. - Int. J. Light Electron Opt. 126, 5963-5970 (2015). https://doi.org/10.1016/i.ijleo.2015.08.109

[18] M. Takeda, H. Ina, and S. Kobayashi, "Fourier-transform method of fringe-pattern analysis for computer-based topography and interferometry", J. Opt. Soc. Am. 72, 156-160 (1982). https://doi.org/10.1364/JOSA.72.000156

[19] E. Cuche, P. Marquet, and C. Depeursinge, "Spatial Filtering for zero-order and twin-image elimination in digital off-axis holography", Appl. Opt. 39, 4070-4075 (2000). https://doi.org/10.1364/A0.39.004070

[20] S. De Nicola, P. Ferraro, A. Finizio, and G. Pierattini, "Wave front reconstruction of Fresnel off-axis holograms with compensation of aberrations by means of phase-shifting digital holography", Opt. Lasers Eng. 37, 331-340 (2002). https://doi.org/10.1016/S0143-8166(01)00087-2

[21] B. Sha, Y. Lu, Y. Xie, Q. Yue, and C. Guo, "Fast reconstruction of multiple off-axis holograms based on a combination of complex encoding and digital spatial multiplexing", Chinese Opt. Lett. 14, 60902 (2016). https://doi.org/10.3788/COL201614.060902

[22] V. Katkovnik, I. A. Shevkunov, N. V Petrov, and K. Egiazarian, "Wavefront reconstruction in digital offaxis holography via sparse coding of amplitude and absolute phase", Opt. Lett. 40, 2417-2420 (2015). https://doi.org/10.1364/OL.40.002417

[23] M. Karray, P. Slangen, and P. Picart, "Comparison between Digital Fresnel Holography and Digital Image-Plane Holography: The Role of the Imaging Aperture", Exp. Mech. 52, 1275-1286 (2012). https://doi.org/10.1007/s11340-012-9604-6

[24] P. Picart, M. Karray, and P. Slangen, "Some Considerations About the Role of the Diaphragm in Digital Image-Plane Holography", DW4C.6 (2012).

[25] E. Sánchez-Ortiga, A. Doblas, G. Saavedra, M. Martínez-Corral, G. Saavedra, and J. Garcia-Sucerquia, "Off-axis digital holographic microscopy: practical design parameters for operating at diffraction limit", Appl. Opt. 53, 2058-2066 (2014).

https://doi.org/10.1364/A0.53.002058 
[26] N. Verrier and M. Atlan, "Off-axis digital hologram reconstruction: some practical considerations", Appl. Opt. 50, H136-H146 (2011). https://doi.org/10.1364/A0.50.00H136

[27] L. Xu, J. Miao, and A. Asundi, "Properties of digital holography based on in-line configuration", Opt. Eng. 39, 3214-3219 (2000). https://doi.org/10.1117/1.1327503

[28] P. Piedrahita-Quintero, R. Castañeda, and J. Garcia-Sucerquia, "Numerical wave propagation in ImageJ", Appl. Opt. 54, 6410-6415 (2015). https://doi.org/10.1364/A0.54.006410

[29] P. Piedrahita, R. Castañeda, and J. Garcia-Sucerquia, http://unal-optodigital.github.io/NumericalPropagation/

[30] R. Castañeda, P. Piedrahita-Quintero, and J. Garcia-Sucerquia, "Image processing and computing for digital holography with ImageJ", Opt. Pura y Apl. 48, 77-84 (2015). https://doi.org/10.1364/A0.54.006410

\section{Introducción}

La reconstrucción numérica de hologramas registrados digitalmente, es un proceso de formación de imágenes conocido como holografía digital (HD), ha generado un fuerte impacto en el mundo científico y tecnológico por uso de medios digitales en reemplazo de materiales fotosensibles holográficos analógicos para el registro de los hologramas y métodos numéricos para reconstruir los hologramas registrados [1]. Desde sus comienzos la HD ha sido utilizada en diversos en campos como la microscopía [2], metrología de sistemas micro electromecánicos [3], el cifrado de información [4], entre muchas otras aplicaciones. La HD al igual que la holografía óptica, pueden entenderse como procesos de formación imágenes en dos pasos, el registro y la reconstrucción. Sin embargo, en la HD el hecho de realizar el registro del holograma de forma digital, permite efectuar operaciones de pre y post procesamiento, ya sea del holograma registrado y/o del holograma reconstruido, que derivan en reconstrucciones que aportan información adicional con referencia a su contraparte óptica y que reducen problemas que la holografía óptica no puede hacer. Por ejemplo, en la HD es posible eliminar en el proceso de reconstrucción los órdenes difractados que no son de utilidad, seleccionando la información del objeto de interés en el espacio de las frecuencias espaciales. La posibilidad de manipular de forma digital de la información registrada facilita el desarrollo de estrategias de mejora del desempeño de la HD. Una de ellas es la reducción del ruido speckle generado por el uso de fuentes coherentes en la etapa de registro, ruido que su contraparte óptica no puede reducirse [5-8].

En este trabajo se analiza el efecto de una de esas posibilidades que el mundo digital le ha aportado a la HD y que le permite filtrar espacialmente el holograma para aislar la imagen a reconstruir. A pesar ser ampliamente conocido por los expertos en holografía digital que la superposición de frecuencias espaciales reduce la calidad de la reconstrucción de los hologramas, no es conocido por estos autores un estudio del efecto del filtrado espacial en la calidad de las reconstrucciones obtenidas a partir de hologramas fuera de eje registrados o no en el límite de difracción. Con el fin de aportar un estudio sistemático inicial en este tema, se hace un breve recuento teórico del proceso de filtrado espacial, seguidamente se muestran los resultados obtenidos y finalmente se explican las conclusiones y aportes de este trabajo.

\section{Análisis teórico}

La holografía digital es una técnica de formación de imágenes que consta de los paso de registro y reconstrucción. En el primero, el patrón de interferencia generado por la superposición en amplitud de la onda objeto y la onda de referencia se registra en una cámara digital; en la Fig. 1 se presenta una ilustración del proceso de registro. La intensidad de este patrón de interferencia, llamado holograma digital, se puede expresar como:

$$
H(x, y)=|R(x, y)+O(x, y)|^{2}
$$


donde $R(x, y)=A_{R} e^{i \varphi_{R}(x, y)}$ es la amplitud compleja de la onda de referencia y $O(x, y)=A_{O}(x, y) e^{i \varphi_{O}(x, y)}$ es la amplitud compleja de la onda objeto. Para finalidades del análisis que se realiza conviene reescribir la ecuación (1) en su forma extensa:

$$
H(x, y)=\left|A_{R}\right|^{2}+|O(x, y)|^{2}+O(x, y) R^{*}(x, y)+O^{*}(x, y) R(x, y) ;
$$

donde* se usa para expresar complejo conjugado. Cuando la ecuación (2) se analiza en el dominio de su transformada de Fourier [9], los dos primeros términos producen el orden cero u orden DC de difracción; el tercer y cuarto término dan origen a los órdenes difractados +1 y -1 , respectivamente, estos términos son portadores de la información de la imagen real e imagen virtual (imágenes gemelas), en ese orden.

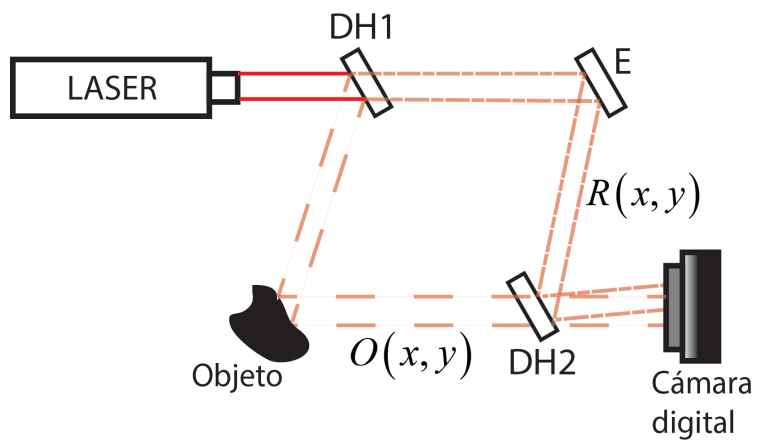

Fig. 1. Diagrama del proceso de registro en holografía digital. DHs Divisores de haz, E espejo, $O(x, y)$ haz objeto y $R(x, y)$ haz de referencia

La segunda etapa que compone el proceso de formación de imágenes mediante la HD consiste en realizar la reconstrucción numérica del holograma digital [10]. El proceso de reconstrucción numérica de hologramas registrados digitalmente consiste en simular el proceso de difracción que sufre la onda de referencia al incidir sobre el holograma registrado. Esta simulación se lleva a cabo calculando por ejemplo la integral de difracción de Fresnel-Kirchhoff [10]

$$
\psi(\xi, \eta, z)=\frac{i}{\lambda} \iint_{-\infty}^{\infty} H(x, y) R(x, y) \frac{\exp (-i k r)}{r} \cos (\Theta) d x d y ;
$$

donde $\psi(\xi, \eta, z)$ representa el campo complejo reconstruido a una distancia z desde el medio de registro, $\lambda$ es la longitud de onda, el termino $r=\sqrt{(x-\xi)^{2}+(y-\eta)^{2}+z^{2}}$ es la distancia entre un punto $(x, y, 0)$ en el plano del medio de registro y un punto $(\xi, \eta, z)$ en el plano de reconstrucción, y finalmente $\Theta$ es el ángulo formado por la dirección perpendicular al plano del holograma con un vector posición que sale del plano del holograma registrado y se dirige al punto de reconstrucción, ver Fig. 2. La ecuación (3) puede ser calculada numéricamente utilizando diferentes formalismos como el espectro angular [11], transformada de Fresnel [12,13], transformada de Fresnel Bluestein [14] o mediante la aproximación de convolución [12]. Estos métodos tienen diferentes rangos de aplicación de acuerdo con la distancia de propagación, el tamaño del pixel del sensor y la longitud de onda[15-17].

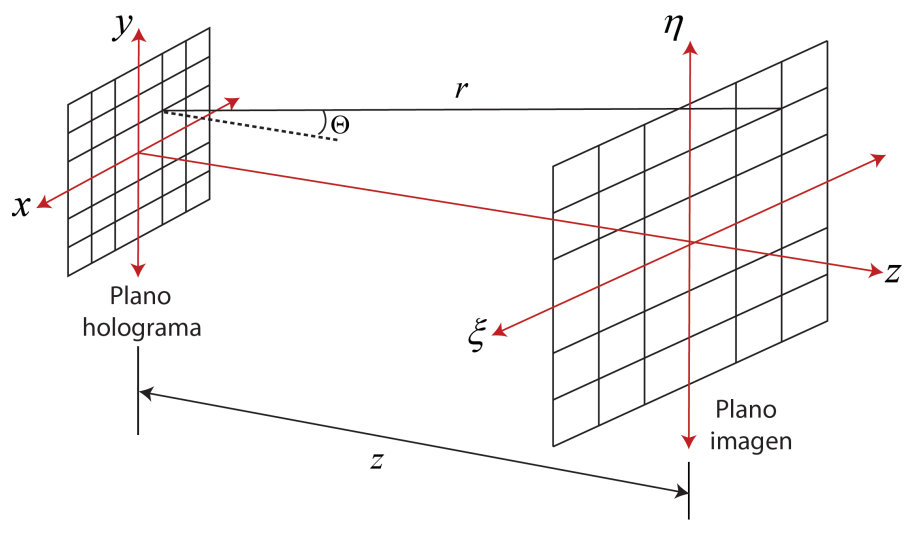

Fig. 2. Esquema del proceso de reconstrucción en holografía digital 
Si bien es posible hacer la reconstrucción del holograma registrado $H(x, y)$ directamente usando el método más adecuado de los mencionados anteriormente, un pre procesamiento sobre el holograma digital permite aislar la información de interés eliminando el orden cero de difracción y una de las imágenes gemelas. Este proceso conocido como filtrado espacial $[18,19]$ permite eliminar los términos adicionales que se consideran como ruido en la reconstrucción numérica y disminuyen considerablemente la calidad del holograma reconstruido. Si el holograma es reconstruido considerando todas las frecuencias espaciales aportadas por todos los órdenes difractados, es decir, sin realizar el filtrado espacial, la imagen obtenida carece de calidad en términos del contraste, debido principalmente a que el orden cero tiene un nivel de intensidad muy superior al de las imágenes gemelas, limitando el uso adecuado del rango dinámico disponible para la representación de las imágenes reconstruidas; esta situación se ilustra en la Fig. 3. El orden cero de difracción con un nivel mayor de intensidad, esencialmente oculta la información de la imagen virtual (desenfocada) y de la imagen real (enfocada) que se alcanza a observar con muy bajo contraste.

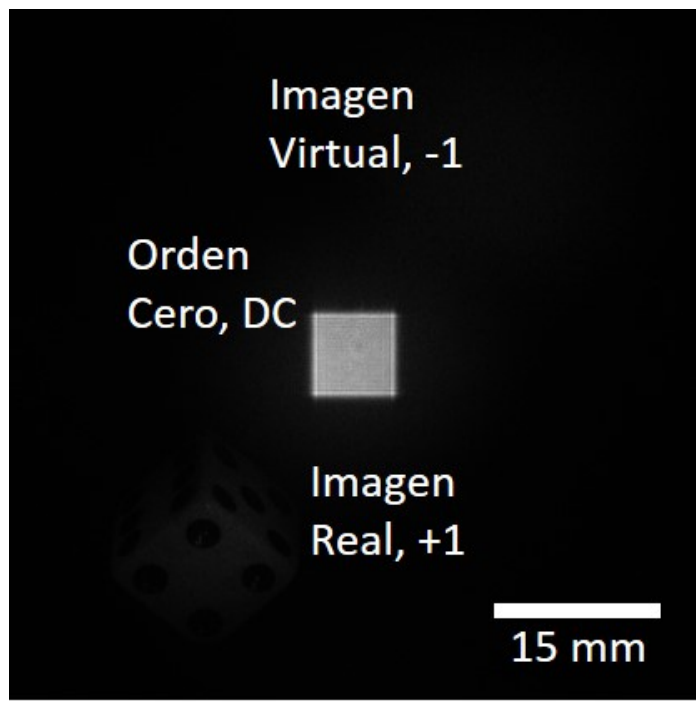

Fig. 3. Reconstrucción numérica de un holograma digital que contiene información de los tres órdenes difractados.

Se han reportado diferentes metodologías que permiten aislar la imagen de interés [19-22], bien sea la correspondiente al orden difractado +1 ó -1 . Sin embargo, el método que es de interés para el análisis realizado es el propuesto por Takeda y conocido con el nombre de filtrado espacial [18]. El método consiste en realizar una selección de las frecuencias espaciales de interés correspondientes al orden +1 ó 1 (en este el artículo se trabajará con las correspondientes al orden +1 ) y eliminar por completo todas las frecuencias espaciales correspondientes a los otros ordenes, es decir, orden DC y la imagen gemela. Con el fin de poder seleccionar exclusivamente las frecuencias portadoras del orden +1 , es necesaria una configuración fuera de eje donde los órdenes difractados puedan separarse por completo y no exista un solapamiento entre ellos. La ubicación de los órdenes difractados es controlada por el ángulo de incidencia que existe entre la onda de referencia y la onda objeto al incidir sobre el plano del medio de registro [23-26] según se ilustra en la Fig. 4.

Si se considera la onda de referencia como una onda plana $R(x, y)=A_{R} e^{i \mathbf{k} r}$ con vector de onda en $z=0$ igual a $\mathbf{k}=2 \pi / \lambda\left(\cos \alpha \mathbf{U}_{\mathbf{x}}+\cos \beta \mathbf{U}_{y}\right)$ donde $\mathbf{U}_{\mathbf{x}}$ y $\mathbf{U}_{y}$ son vectores unitarios a lo largo de los ejes $x$ e $y$, respectivamente, la ubicación de los órdenes difractados +1 y -1 en el espacio de las frecuencias está controlada por los valores de los ángulos $\alpha$ y $\beta$; si estos ángulos son iguales a cero se tiene una configuración de holografía en línea [27], donde todos los órdenes difractados se solapan en el centro del espacio de las frecuencias. Por otra parte, si los ángulos son diferentes de cero, la configuración es fuera de eje. En la Fig. 5 se ilustra la ubicación de los órdenes difractados en el espectro de Fourier de hologramas registrados fuera de eje para diferentes valores de los ángulos $\alpha$ y $\beta$. En esta figura, el orden cero es generado por el término $D C=\mathfrak{I}\left\{\left|A_{R}\right|^{2}+|O(x, y)|^{2}\right\}, \mathfrak{I}\left\{O R^{*}\right\}$ y $\mathfrak{I}\left\{O^{*} R\right\}$ corresponden a la transformada de Fourier de los órdenes +1 y -1 , respectivamente. Mientras que en la Fig. 5 (a) el valor de estos ángulos no es suficiente para separar los órdenes difractados, en (b) se muestra como con la adecuada selección del ángulo entre la ondas de referencia y objeto se puede garantizar que no exista solapamiento entre los 
órdenes difractados haciendo uso correcto del espacio ancho de banda; en esta condición se obtiene una configuración de HD fuera de eje operando en el límite de difracción [25,26].

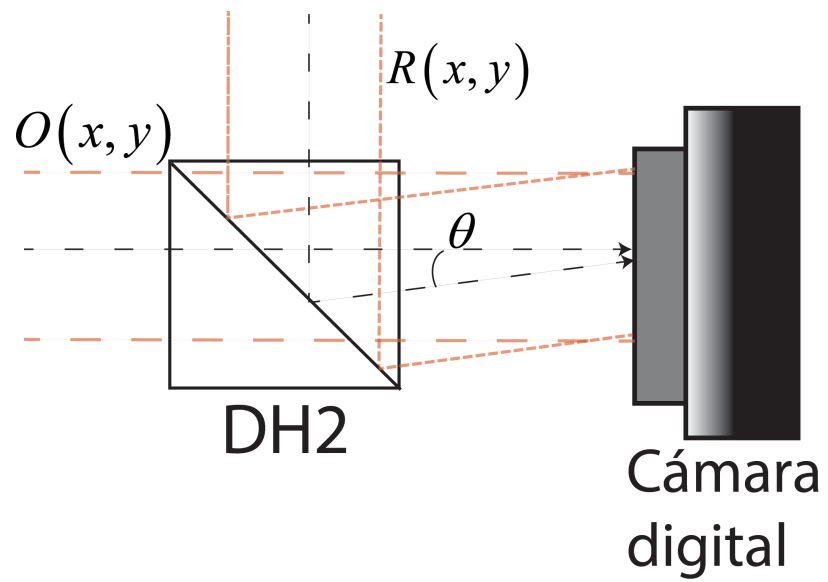

Fig. 4. Representación gráfica del ángulo de incidencia entre la onda de referencia y la onda objeto en holografía digital. $O(x, y)$ es la onda objeto, $R(x, y)$ es la onda de referencia, DH2: divisor de haz y finalmente $\theta$ es el ángulo entre $O(x, y)$ y $R(x, y)$.
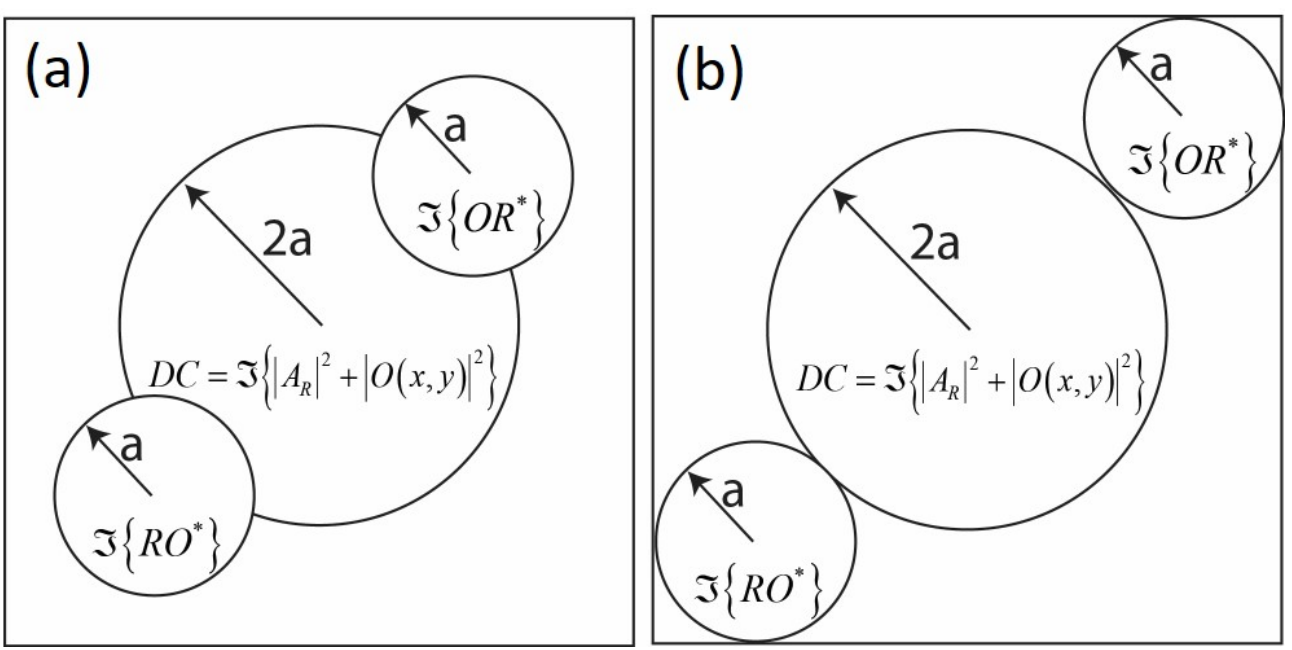

Fig. 5. Representación del espectro de Fourier de hologramas registrados en configuración fuera de eje. En (a) el registro no opera en el límite de la difracción produciendo solapamiento de los órdenes difractados y (b) el holograma es registrado en el límite de difracción. El orden DC se encuentra localizado en el centro del espectro y su dimensión es el doble a las dimensiones de los órdenes difractados +1 y -1

Puesto que el método utilizado en el este trabajo realiza el filtrado espacial en el dominio de Fourier, el primer paso de este proceso es la realización de la transformada de Fourier del holograma registrado $H(x, y)$, es decir:

$$
\begin{aligned}
\boldsymbol{F}\{H(x, y)\} & =\tilde{H}(u, v) \\
& =D C+A_{R}^{*} \tilde{O}(u, v) \otimes \delta\left(u+\frac{\cos \alpha}{\lambda}, v+\frac{\cos \beta}{\lambda}\right)+A_{R} \tilde{O}^{*}(u, v) \otimes \delta\left(u-\frac{\cos \alpha}{\lambda}, v-\frac{\cos \beta}{\lambda}\right) ;
\end{aligned}
$$

donde $\otimes$ es el operador de convolución y $\delta$ representa la función delta de Dirac. Una vez se tienen los órdenes difractados en el espacio de las frecuencias, se realiza la selección, por ejemplo del orden +1 , y se almacenan en una nueva transformada del holograma $\tilde{H}_{F}(u, v)$ :

$$
\tilde{H}_{F}(u, v)=A_{R}^{*} \tilde{O}(u, v) \otimes \delta\left(u+\frac{\cos \alpha}{\lambda}, v+\frac{\cos \beta}{\lambda}\right) ;
$$

La ecuación (5) indica que las frecuencias del orden +1 se encuentran desplazadas del centro de espacio de las frecuencias en un cantidad determinado por los ángulos $\alpha$ y $\beta$. Ahora se realiza un desplazamiento 
hacia el centro del especio de las frecuencias del orden +1 , eliminando la dependencia del orden difractado de dichos ángulos. Después de realizar el centrado del orden difractado se toma la transformada inversa de Fourier, para obtener de esta manera un holograma digital filtrado en el plano de la cámara digital:

$$
H_{F}(x, y)=A_{R} O(x, y) \text {; }
$$

La Fig. 6 es una representación del proceso de filtrado espacial. En el panel (a) se muestra el holograma registrado descrito por la ecuación (1), en el panel (b) se aprecia el espectro de la transformada de Fourier del holograma, ecuación (4); en esta imagen se observan los tres órdenes difractados. La correcta separación de estos órdenes permite seleccionar las frecuencias espaciales del orden +1 , (región encerrada en blanco). Una vez se ha realizado la selección de las frecuencias de interés se almacenan y se eliminan por completo el resto de frecuencias espaciales. Las frecuencias almacenadas se llevan al centro del espectro de Fourier, como se muestra en el panel (c) y el panel (d) corresponde a la transformada inversa de Fourier del panel (c), obteniendo finalmente el nuevo holograma filtrado, representado por la ecuación (6).

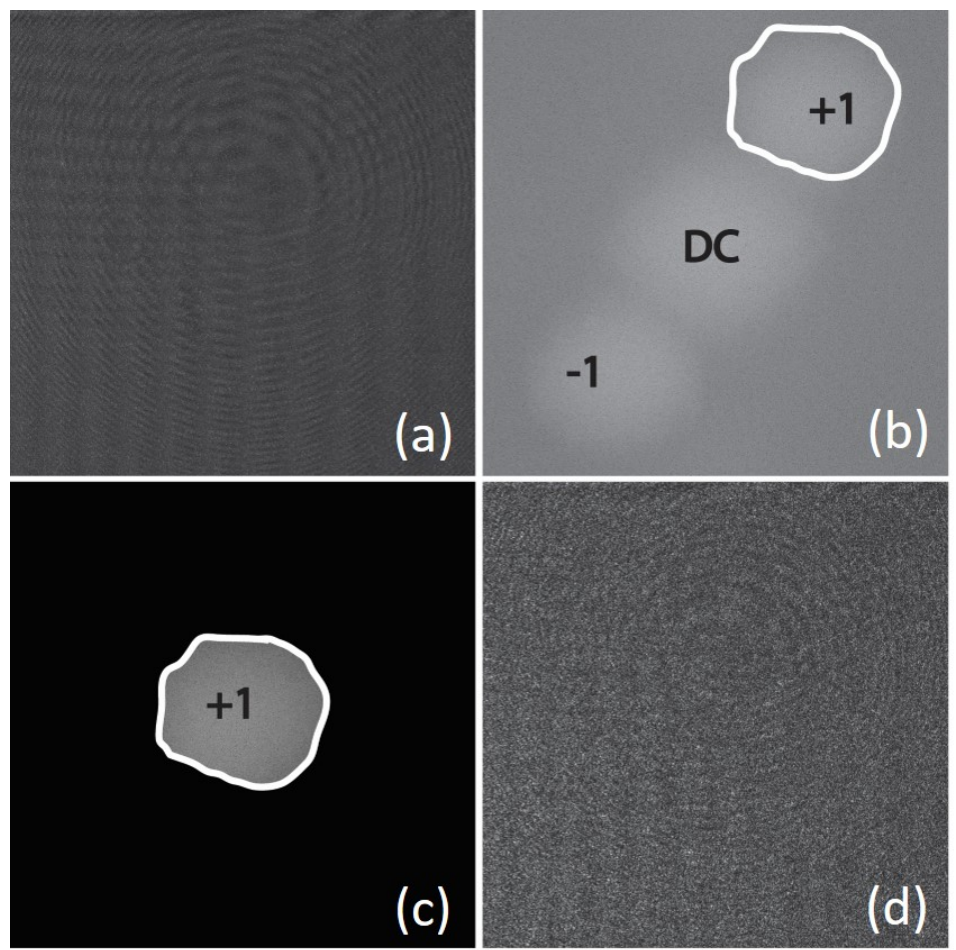

Fig. 6. Esquema del proceso de filtrado espacial. (a) holograma registrado, (b) espectro de Fourier del holograma registrado, (c) orden de difracción +1 centrado en el espectro de Fourier y (d) holograma filtrado espacialmente.

La ecuación (6) indica que la onda difractada por el objeto $O(\mathrm{x}, \mathrm{y})$ puede ser recuperada en el plano de la cámara digital por medio de la multiplicación del holograma digital filtrado por la amplitud de la onda de referencia, la cual por simplicidad puede ser considerada como unitaria. Este campo complejo puede ser propagado a cualquier plano para obtener la información difractada por el objeto. Si el plano de propagación corresponde al plano de ubicación del objeto, se obtendrá una imagen del objeto.

\section{Resultados experimentales.}

La Fig. 7 muestra la configuración óptica utilizada durante el proceso de registro de los hologramas fuera de eje adquiridos experimentalmente. Como fuente de iluminación se usó un láser de He-Ne de longitud de onda de $632.8 \mathrm{~nm}$. El haz de luz proveniente del láser es dividido en el divisor de haz (DH1) para producir el haz de referencia $R(\mathrm{x}, \mathrm{y})$ y el haz objeto $O(\mathrm{x}, \mathrm{y})$. El haz de referencia se convierte en una onda plana luego de pasar un sistema colimador formado por una el objetivo de microscopio 2 (OM2) y una lente (L). Por otra parte, el haz objeto incide sobre el objetivo de microscopio 1 (OM1) y luego sobre el objeto; la luz reflejada por el objeto se combina mediante el divisor de haz 2 (DH2) con la onda plana de referencia y 
generan un patrón de interferencia el cual es almacenado en el medio de registro digital. Los hologramas fueron registrados mediante una cámara digital CMOS (PixeLink ®-PL-D725MU-T) con 2592 x 2048 pixeles cuadrados de 4.8 um de lado. La cámara fue ubicada a $70 \mathrm{~cm}$ del objeto.

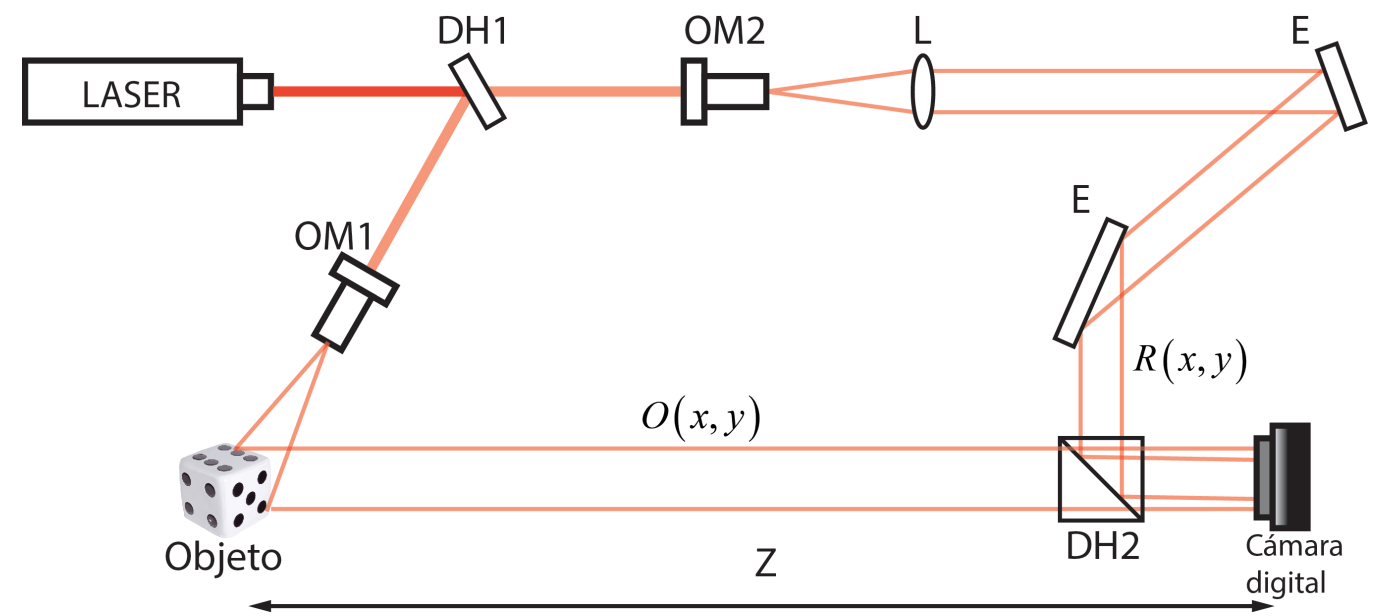

Fig. 7. Ilustración del montaje óptico usado para el registro de los hologramas en fuera de eje. Ver texto para detalles.

Con el fin mostrar la importancia que tienen sobre la reconstrucción numérica de un holograma digital el correcto filtrado espacial y su estrecha relación con el registro del holograma, se muestra a continuación una serie de casos que corresponden a hologramas reconstruidos en condiciones inadecuadas. Se muestra la reconstrucción de hologramas registrados fuera del límite de difracción o de hologramas operando en el límite de difracción pero con una selección errónea de las frecuencias espaciales. El caso final ilustra cómo es posible seleccionar de manera apropiada las frecuencias espaciales para una correcta reconstrucción cuando el holograma se registra en el límite de difracción. Todos los procesos digitales requeridos se llevaron a cabo con el plugin Numerical wave propagation [28-30], el cual fue desarrollado por el Grupo de óptica y procesamiento opto-digital de la Universidad Nacional de Colombia sede Medellín.
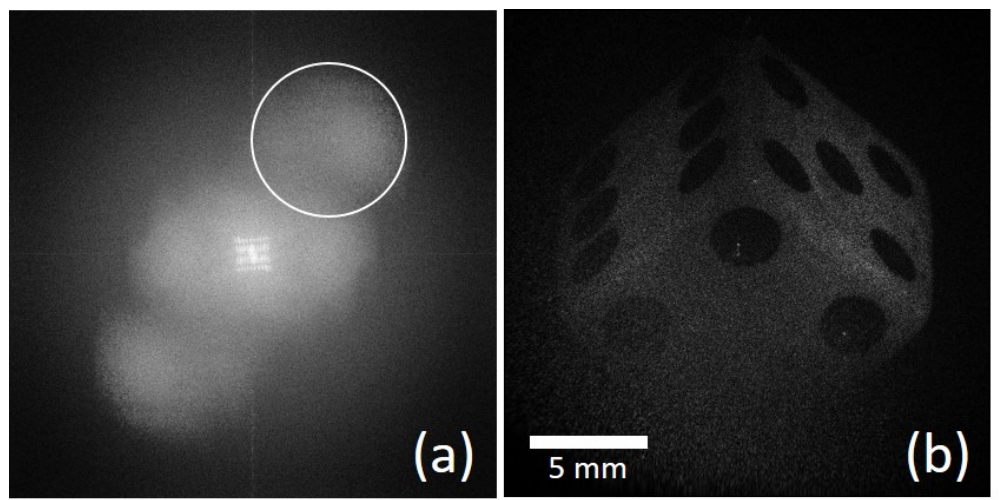

Fig. 8. Reconstrucción numérica de un holograma registrado fuera del límite de difracción. Panel (a) espectro de Fourier del holograma registrado y panel (b) es la reconstrucción obtenida para las frecuencias seleccionadas en el círculo blanco del panel (a).

La Fig. 8 muestra los resultados obtenidos al reconstruir numéricamente un holograma registrado fuera del límite de difracción. Como se indicó anteriormente, en esta configuración se produce la superposición de los órdenes difractados +1 y -1 con el término DC, haciendo imposible realizar un correcto proceso de filtrado espacial, debido a que no es posible aislar totalmente las frecuencias espaciales que corresponden a alguna de las imágenes gemelas. En este caso siempre estarán presentas frecuencias provenientes del orden DC en la reconstrucción del objeto. El panel (a) corresponde al espectro de la transformada de Fourier del holograma, donde se puede notar que los órdenes difractados +1 y -1 se solapan con el orden cero. El panel (b) es la reconstrucción obtenida luego de seleccionar las frecuencias del orden +1 , región encerrada en el círculo de color blanco que se muestra en (a). De la reconstrucción se puede notar que la imagen carece de calidad, debido a la presencia de información del orden cero, lo cual genera sobre el 
objeto ruido que afecta su visibilidad. Estos resultados indican que si existe solapamiento de los órdenes difractados, es decir si los hologramas no son registrados en el límite de difracción, no es posible realizar un filtrado espacial que aísle de forma exclusiva el orden difractado de interés (en este ejemplo orden +1 ) sin perturbación de la información en el orden cero de difracción y se pueda realizar una reconstrucción de la imagen sin perturbación. Por esta razón nos limitamos en adelante a estudiar los efectos del filtrado espacial operando en hologramas digitales registrados en el límite de difracción.

En la Fig. 9, se muestran los efectos de la realización de filtrados espaciales que no consideran las frecuencias espaciales adecuadas de un holograma registrado en el límite de difracción. En el panel (a) de la Fig. 9 se ilustra el espectro de la transformada de Fourier del holograma registrado en el límite de difracción; como se puede observar los órdenes difractados no se superponen y existe una separación clara entre ellos. En los paneles (b) y (c) se muestran las reconstrucciones numéricas después de realizar el proceso del filtrado espacial seleccionando las frecuencias espaciales encerradas en el círculo de línea discontinua y el círculo de línea solida del panel (a), respectivamente. Como puede notarse en los paneles (b) y (c), ambas reconstrucciones distan de una imagen del dado con calidad óptima, ya sea por falta de información (b) o por la aparición de ruido (c) similar al caso del registro del holograma fuera del límite de difracción. En panel (b), la imagen del dado se ve recortada debido a la selección incompleta de las frecuencias espaciales que la componen. Si por el contrario el filtrado espacial incluye frecuencias espaciales correspondientes al orden cero, estas frecuencias generan ruido superpuesto sobre la imagen reconstruida, de forma completamente similar al caso del registro fuera del límite de difracción donde es imposible aislar completamente las frecuencias espaciales que componen la imagen de interés.
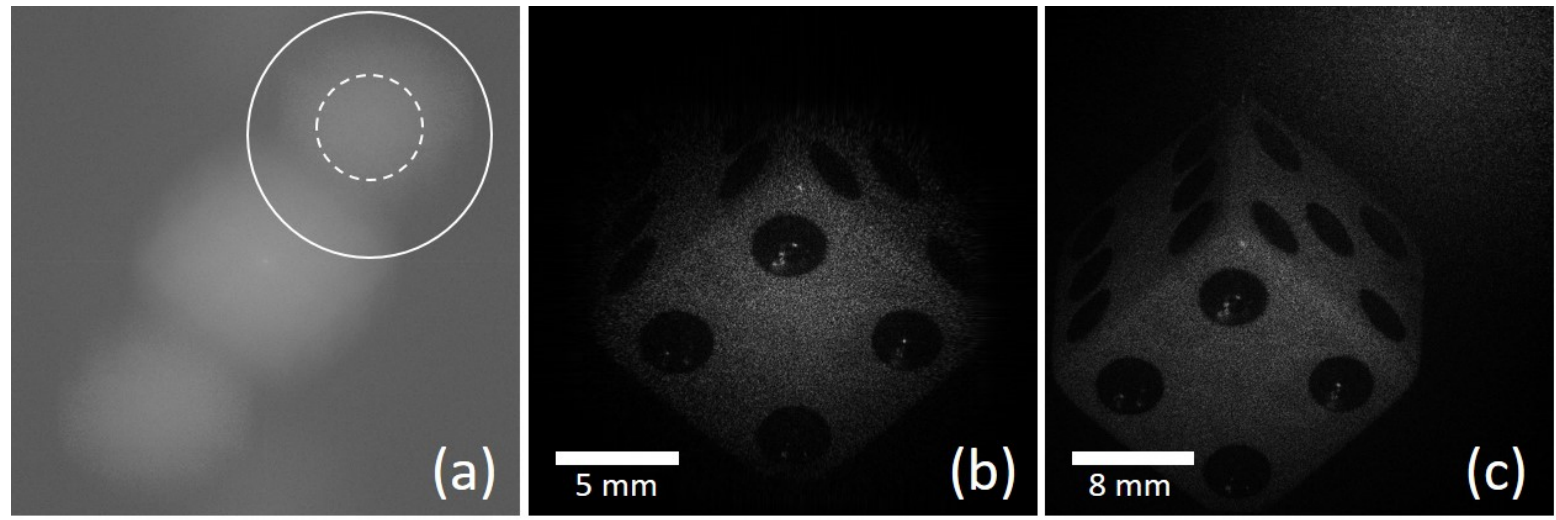

Fig. 9. Reconstrucción numérica de un holograma registrado en el límite de difracción, cuando se realiza de forma inadecuada el filtrado espacial. (a) Espectro de Fourier, (b) reconstrucción obtenida después de seleccionar las frecuencias espaciales del círculo de línea discontinua de (a) y finalmente en (c) imagen reconstruida después seleccionar las frecuencias espaciales del círculo de línea solida de (a)
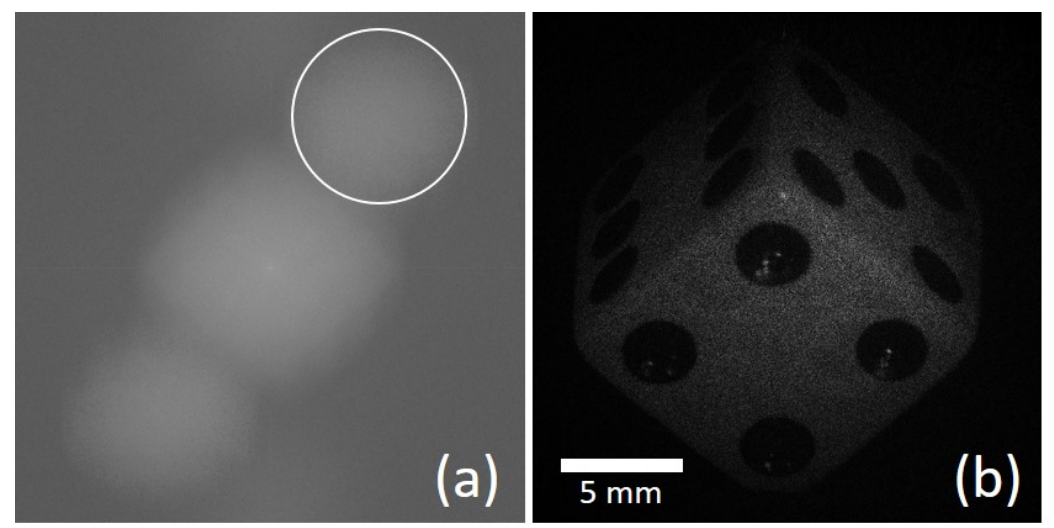

Fig. 10. Reconstrucción numérica del mismo holograma de la Fig.2 después de realizar el correcto proceso de filtrado espacial. (a) espectro de Fourier del holograma registrado en el límite de difracción y (b) imagen reconstruida realizando el filtrado espacial de las frecuencias seleccionadas en el círculo blanco del panel (a) 
Si la selección de las frecuencias espaciales que componen la imagen a reconstruir es realizada de forma adecuada como se ilustra en la Fig. 10 (a), se obtienen los resultados mostrados en el panel (b) de esta figura. En el espectro de Fourier del holograma, panel (a), se seleccionan las frecuencias que corresponden de forma exclusiva al orden de difracción +1 por medio del filtro ilustrado por el circulo blanco de este panel. Sobre estas frecuencias se realiza la operación del filtrado espacial para conducir a la reconstrucción del panel (b). Puede notarse de la reconstrucción que la selección adecuada de las frecuencias espaciales conduce a una imagen completa del objeto de interés, además de estar libre de ruido originado por la inclusión de frecuencias espaciales pertenecientes al orden cero de difracción.

Los resultados de la Fig. 10, indican que la correcta reconstrucción en HD está condicionada por el registro del holograma en el límite de difracción y la selección correcta de las frecuencias espaciales que componen la imagen de interés, únicamente posible si los órdenes difractados están completamente separados en el espectro de Fourier del holograma registrado.

\section{Conclusiones}

En este trabajo se ha presentado un análisis de la relevancia que tiene en la reconstrucción numérica de hologramas registrados digitalmente en configuración fuera de eje, el registro de los hologramas fuera o dentro del límite de difracción y el correcto filtrado espacial. Los resultados permiten concluir que si el holograma no está registrado en el límite de difracción es imposible obtener una reconstrucción numérica adecuada, ya que siempre se incluirá información del orden cero en las frecuencias espaciales correspondientes al orden difractado de interés. El registro fuera del límite de difracción conduce al solapamiento de los órdenes +1 y -1 con el orden DC, lo cual reduce la calidad de la imagen del objeto reconstruido por la introducción de ruido sobre la imagen reconstruida.

Por otra parte, los resultados también permiten concluir que no es suficiente con que el holograma sea adquirido en el límite de difracción. Para la reconstrucción adecuada se requiere una correcta selección de las frecuencias espaciales de la imagen que se quiere reconstruir. Por ejemplo, seleccionar en el proceso de filtrado espacial una región que incluya frecuencias del orden DC así no exista solapamiento con los otros ordenes difractados, reduce la calidad del objeto reconstruido por la generación de ruido sobre la imagen del objeto. Ahora, para el caso en que las frecuencias seleccionadas no corresponden en su totalidad al orden difractado +1 o -1 , se deja por fuera información de la imagen del objeto, ocasionando como resultando una reconstrucción numérica que presenta una imagen incompleta del objeto incompleto.

La correcta reconstrucción numérica de hologramas registrados digitalmente en configuración fuera de eje, está condicionada por el registro del holograma que garantice el no solapamiento de los órdenes difractados y la selección correcta de las frecuencias espaciales que componen la imagen de interés. Estas condiciones son posibles de alcanzar únicamente si los órdenes difractados están completamente separados en el espectro de Fourier del holograma registrado, es decir, si el holograma está registrado en el límite de difracción.

\section{Agradecimientos}

Los autores agradecen el soporte brindado por la Universidad Nacional de Colombia, mediante el programa de Jóvenes Investigadores de Colciencias código Hermes 28751, la Convocatoria Nacional de Proyectos para el Fortalecimiento de la Investigación de la Universidad Nacional de Colombia 2016-2018 código Hermes 35765 y al Instituto Tecnológico Metropolitano ITM número de proyecto P14217. 